\title{
Synergistic Action of Copper Catalysts and Lewis Acids in Carbon-Carbon Bond Forming Reactions
}

\author{
Manas Das and Syuzanna Harutyunyan*
}

\begin{abstract}
Combining Lewis acids with copper catalysis and highly reactive Grignard reagents allows fine tuning of the reactivity and selectivity of both inherently unreactive and highly reactive electrophilic substrates in asymmetric $\mathrm{C}-\mathrm{C}$ bond forming reactions. In this review we will discuss various examples where the application of this strategy provides access to efficient catalytic processes for the synthesis of chiral molecules such as alcohols, amides and heteroarenes.
\end{abstract}

Keywords: Alkylation · Asymmetric catalysis - Copper · Grignard reagents · Lewis acid

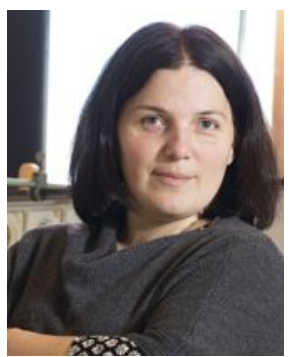

Syuzanna
Harutyunyan
received her Master's
degree in Chemistry
from Yerevan State
University, Armenia,
after which she
moved to Moscow,
Russia, in 1999
to undertake her $\mathrm{PhD}$ studies under the supervision of Prof. Belokon. After spending several months in 2002 as a visiting scientist in Warsaw, Poland, working with Prof. Grela, Syuzanna joined the research group of Prof. Feringa at the University of Groningen, the Netherlands, in 2003 as a postdoctoral Research Fellow. In 2007 Syuzanna joined the Process\&Development department at Janssen Pharmaceutica, Belgium. Syuzanna started her independent research career in 2010, when she was appointed as a tenure-track Assistant Professor at the University of Groningen. In 2013 she was tenured and promoted to Associate Professor and in 2018 to full professor at the University of Groningen. Syuzanna's research activities include synthesis, organometallic reactions, catalysis, enantioselective transformations and autocatalysis.

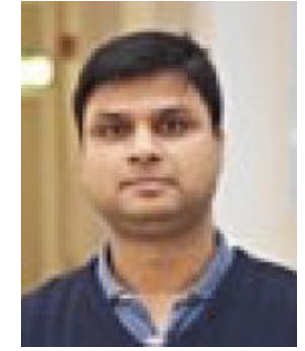

\section{Manas Das}

obtained his master of science in chemistry from Indian Institute of Technology Guwahati, India. Then he moved to University College Dublin, Ireland to pursue his doctoral studies with Prof. Donal F. O'Shea. After getting his $\mathrm{PhD}$ in January 2015 he joined Royal College of Surgeons in Ireland as a postdoctoral researcher. After spending couple of years, in January, 2017 he moved to Institut für Chemie, Technische Universität Berlin, for his next postdoc with Prof. Dr. Johannes Teichert. In the beginning of 2018, he joined the group of Prof. Dr. Syuzanna R. Harutyunyan in the University of Groningen and is presently working on asymmetric copper-catalysis.

\section{Introduction}

The industrial production of single enantiomers of chiral therapeutics and fine chemicals still relies predominantly on non-catalytic, stoichiometric synthetic methods and the use of chiral separation approaches. ${ }^{[1]}$ A shortage of relevant catalytic enantioselective transformations is at the core of these persistent difficulties in preparing chemicals as single enantiomers. ${ }^{[2]}$ The challenge is in finding catalytic transformations that simultaneously achieve high levels of stereo-, regio- and chemoselectivity, as this avoids the accumulation of unnecessary steps, minimizes the reliance on protecting groups, and contributes to both atom- and step-economy.[1,2] These types of selectivity control, and in particular stereoselectivity, are especially relevant for drug discovery and the pharmaceutical industry, because complex, chiral molecules are more likely to show useful bioactive properties than achiral, flat compounds.[3] In this context catalytic asymmetric carbon-carbon bond formation is the most straightforward approach for the preparation of chiral molecules. ${ }^{[4]}$ An important area of research in this field, and one that our group in recent years has been actively engaged in, is catalytic asymmetric addition of organometallic reagents to activated $\mathrm{C}=\mathrm{C}, \mathrm{C}=\mathrm{O}$ and $\mathrm{C}=\mathrm{N}$ bonds. ${ }^{[4-6]}$ In 2011 we reported the first example of a catalytic asymmetric Grignard reaction enabled by chiral copper-diphosphine-based catalysts, which promotes the addition of Grignard reagents to aryl alkyl ketones, resulting in various chiral $\alpha$-tertiary alcohols (Scheme 1). ${ }^{[7,8]}$

In these reactions copper catalysis was sufficient to outcompete the non-catalysed direct addition of Grignard reagent to ketones while simultaneously providing stereocontrol. However, when attempting to extend this catalytic system to acyl silanes we were faced with considerably more difficulties. ${ }^{[9]}$ Apart from problems with non-catalysed alkylations of acyl silanes, the Meerwein-Ponndorf-Verley (MPV)

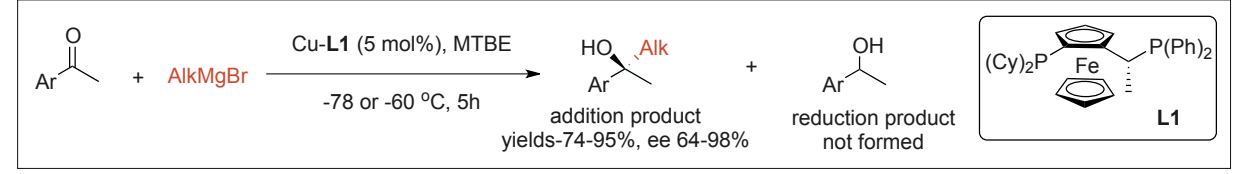

Scheme 1. Copper(I)-catalyzed asymmetric addition of Grignard reagents to aryl-alkyl ketones. 
type reduction ${ }^{[10,11]}$ of the corresponding substrates was found to be the main issue affecting this chemistry (Scheme 2a). Assuming that the reduction reaction is a result of the activation of the $\mathrm{C}=\mathrm{O}$ moiety through coordination with the magnesium ion of the Grignard reagent followed by $\beta$-hydride transfer, we predicted that MPV type reduction could be avoided by using Lewis acid (LA) additives (Scheme 2a, b).

Although LA activation of various reagents towards nucleophilic addition is a powerful tool that has become common practice in organic synthesis, ${ }^{[12-14]}$ the crucial prerequisite for the viability of our strategy was the compatibility of LA with the Grignard reagent. During our research we identified LAs that are compatible with Grignard reagents and employed them in two distinct ways: for activation of electrophilic substrates towards nucleophilic attack, and for prevention of side reactions by using the LA as an in situ protecting group. These two modes of action of LAs, combined with their compatibility and synergy with copper catalysts and Grignard reagents, form the central theme of this review.

\section{Lewis Acids as in situ Protecting Groups}

\subsection{Copper(I)-catalysed Enantioselective Addition of Grignard Reagents to Acylsilanes}

Chiral $\alpha$-silylated alcohols are important building blocks in stereoselective carboncarbon bond formation and rearrangement reactions. ${ }^{[15,16]}$ Whereas several methods are available that provide access to enantiopure secondary $\alpha$-silyl alcohols, ${ }^{[17-19]}$ a general catalytic method to synthesise tertiary $\alpha$-silyl alcohols ${ }^{[20]}$ did not exist until recently. The most straightforward approach would be the catalytic asymmetric addition of organometallic reagents to acylsilanes. The main hurdle that must be overcome to achieve this consists of a single, formidable competing reaction, namely a non-catalytic MPV which leads to the formation of racemic secondary $\alpha$-silyl alcohols. For example, during their attempted alkylation of acylsilanes with diethylzinc the Xu group documented that the reduction of the carbonyl moiety was the major pathway. ${ }^{[21]}$

The $\mathrm{Cu}(\mathrm{I})$-catalysed Grignard reaction methodology that had been developed by our group previously for additions to aryl alkyl ketones (Scheme 1) ${ }^{[8]}$ was applied to acylsilanes. ${ }^{[9]}$ Although the desired $\alpha$-silylated alcohol 2a was obtained with high $90 \%$ ee, the major product of the reaction corresponded to racemic secondary $\alpha$-silyl alcohol 3 derived from MPV type reduction of the acylsilane (Scheme 3, entry 1). Variation of the common reaction parameters, namely the chiral ligands, solvents, and temperature, did not have a significant effect on the chemoselectivity of the reaction. Presuming that the reduction of the carbonyl group is a result of a $\beta$-hydride transfer from the Grignard reagent, we hypothesised that a LA could serve as an in situ protecting group for the carbonyl moiety and allow the catalytic addition pathway to outcompete the undesired reduction pathway. With the compatibility of LAs and Grignard reagents in mind, initial screening was focused on mild LAs $\left(\mathrm{MgBr}_{2}, \mathrm{TMSCl}\right)$, which unfortunately did not improve the chemoselectivity. However, in the presence of $\mathrm{CeCl}_{3}$, commonly used to promote 1,2-additions, both the chemoselectivity and enantioselectivity improved significantly (Scheme 3, entry 3 ). Possible participation of organocerium species in the addition was excluded by the experiment in which only starting material was recovered when isobutylcerium ${ }^{[22]}$ was employed for the alkylation reaction under copper catalysis.

Borane-based LAs play key roles as catalysts and activators in organic synthesis, ${ }^{[12,23]}$ and despite their potential to react with the Grignard reagent these LAs were also explored in this chemistry. Interestingly, with $\mathrm{BF}_{3} \cdot \mathrm{OEt}_{2}$ an improved chemoselectivity, with a 3:1 addition to reduction ratio, was observed, but at the cost of a decrease in the ee to $86 \%$ (Scheme 3 , entry 4). Remarkably, a 1:1 mixture of $\mathrm{BF}_{3} \cdot \mathrm{OEt}_{2} / \mathrm{CeCl}_{3}$ resulted in a dramatic decrease in reduction (entry 5), and provided both a high chemo- (5:1, carbonyl addition/MPV reduction) and enantioselectivity $(90 \%)$. With these conditions in hand we explored the scope of the reaction and found that various substituted aryl acylsilanes and vinyl acylsilanes can be used in combination with alkyl Grignard reagents to provide the addition products with ex- cellent results (for the selected scope see Scheme 3). Furthermore, for vinyl acylsilanes a mere 0.25 equiv of LAs is already sufficient to achieve high yields and enantioselectivities for addition products $\mathbf{2 d - 2 f}$.

\subsection{Copper(I)-catalysed Enantioselective Addition of Grignard Reagents to Diaryl Ketones}

Another important class of compounds that can be accessed via addition of organometallics to the corresponding carbonyl precursors is formed by diarylmethanols and aryl heteroarylmethanols, structural motives extensively present in drugs. ${ }^{24]}$ Unfortunately, with the main focus on arylations, ${ }^{[25]}$ the low reactivity, the similarity of the enantiotopic faces of bisaromatic ketones, as well as their steric bulk, have prevented the development of efficient catalytic asymmetric alkylation reactions. In addition to this set of problems, due to the increased steric bulk of the substrate, MPV-type reduction of diaryl ketones is another major issue affecting this chemistry, similarly as was shown to be the case for acylsilanes. Following the success of the copper-catalysed alkylation of acylsilanes, a chiral $\mathrm{Cu}(\mathrm{I})$-diphosphinebased catalytic system was employed for the alkylation of various aryl heteroaryl ketones. ${ }^{[26]}$ Also in this case the use of a mixture of $\mathrm{LAs}$ and $\mathrm{BF}_{3} \cdot \mathrm{OEt}_{2} / \mathrm{CeCl}_{3}(1: 1)$ was required to improve the reactivity and to outcompete the undesired reduction via MPV-type reaction (Scheme 4).

Although this represents the first example of a direct catalytic asymmetric alkylation of diaryl ketones with enantioselectivities of up to $69 \%$, the major drawback of the methodology is the poor product yield. The low stability of both the alkoxide and the corresponding diarylmethanol leads to
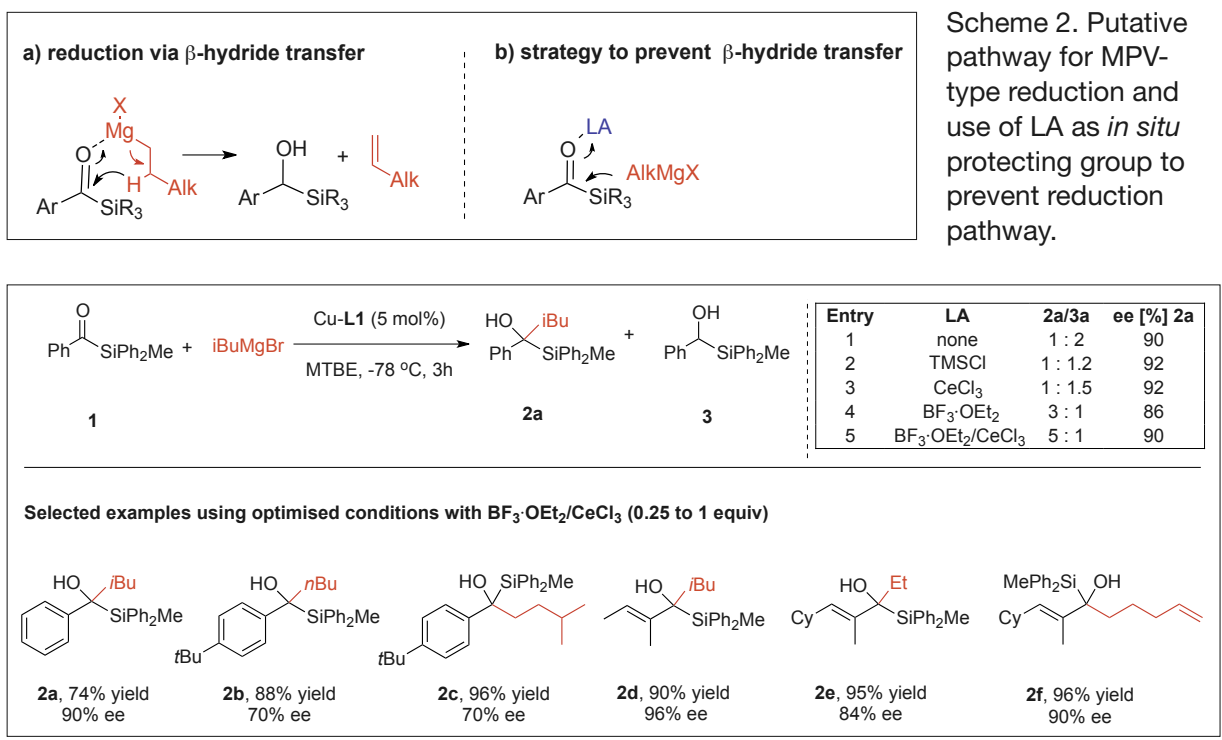

Scheme 3. Representative examples of catalytic asymmetric addition of Grignard reagents to acylsilanes showcasing the key role of the $\mathrm{LA}$ mixture $\left(\mathrm{BF}_{3} \cdot \mathrm{OEt}_{2} / \mathrm{CeCl}_{3}\right)$. 
the formation of the side product 7 from dehydration during the reaction and purification. Therefore this new approach needs improved yields and ees to become a viable method for accessing pharmaceutically valuable molecules.

\section{Lewis Acids for Activation of Inherently Unreactive Substrates}

\subsection{Copper(I)-catalysed \\ Enantioselective Addition of Grignard Reagents to Alkenyl-N- heteroarenes}

Catalytic additions of hard organometallics to $\alpha, \beta$-unsaturated carbonyl derivatives forge $\mathrm{C}-\mathrm{C}$ bonds and rank among the most fundamental reactions in chemical synthesis. ${ }^{[4,27]}$ In this context, alkenyl- $N$-heteroarenes can be formally considered as Michael acceptors, [28,29] but application of these compounds in this way has been limited so far, despite the potential of accessing chiral heteroaromatic compounds that can be of interest for medicinal chemistry. ${ }^{[30]}$ The sporadic reports in this area are restricted to precious metal-catalysed arylations. ${ }^{[31-33]}$ This lack of methodologies is the result of the intrinsically low reactivity of alkenyl-heteroarenes towards nucleophilic additions when compared to common carbonyl-based Michael acceptors. In contrast, chiral $\mathrm{Cu}(\mathrm{I})$-based catalysts permit enantioselective additions of highly reactive alkyl Grignard reagents to various reactive
Michael acceptors, such as $\alpha, \beta$-unsaturated ketones and esters, and thioesters. ${ }^{[34-36]}$

We decided to take advantage of the high reactivity of Grignard reagents and apply them in asymmetric conjugate additions (ACA) to alkenyl-heteroarenes. ${ }^{[37]}$ The first attempts of alkylation using Grignard reagents and chiral $\mathrm{Cu}$-based catalysts resulted in complex mixtures at temperatures above $20{ }^{\circ} \mathrm{C}$ and in no product formation at temperatures below $-50{ }^{\circ} \mathrm{C}$, thus highlighting the low reactivity issues of alkenyl-heteroarenes. However, activation of electrophilic substrates toward nucleophilic addition is the most common use of LAs, and from our work with acylsilanes, where a LA mixture was used to promote the addition over the reduction pathway, we learned that LAs are compatible with both chiral copper catalysts and Grignard reagents, as long as reactions are carried out at temperatures below $-50{ }^{\circ} \mathrm{C}$.

We anticipated that coordination of a strong LA to the $N$-atom of the heteroarene should significantly enhance the electrophilicity of the adjacent olefinic moiety towards nucleophilic addition, thus representing a viable strategy for overcoming the reactivity issues associated with alkenyl-heteroarenes. Scrutiny of the reaction conditions revealed that Grignard reagents can indeed be added enantioselectively to alkenyl heteroarenes in the presence of $\mathrm{BF}_{3} \cdot \mathrm{OEt}_{2}$ as a LA and chiral copper-diphosphine complex as a catalyst (Scheme 5). It is noteworthy that, unlike for the acylsilanes,

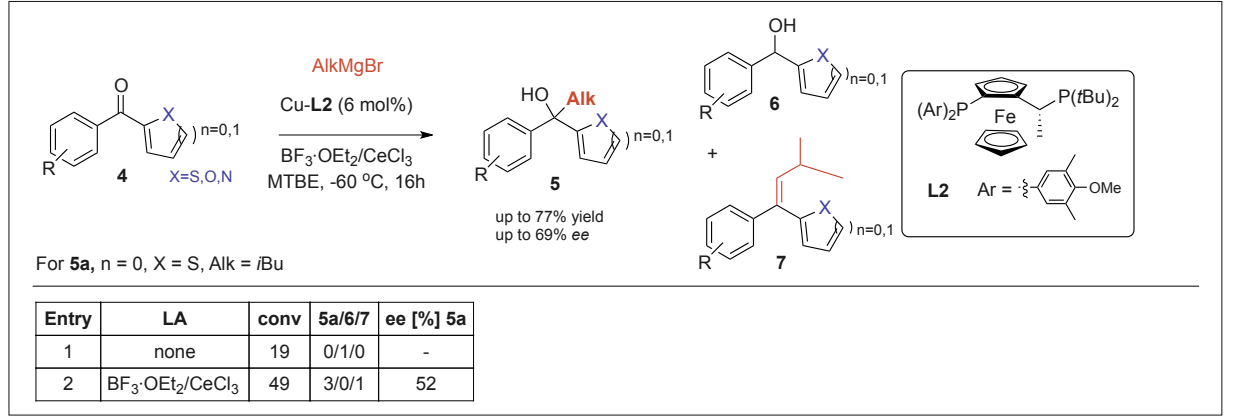

Scheme 4. Catalytic asymmetric alkylation of diaryl ketones with Grignard reagents in the presence of the LA mixture $\left(\mathrm{BF}_{3} \cdot \mathrm{OEt}_{2} / \mathrm{CeCl}_{3}\right)$. a mixture of LAs is not required for the heteroarene chemistry. This methodology allowed for the first time the introduction of linear, branched, and cyclic alkyl chains at the $\beta$-carbon position of alkenyl heteroarenes with excellent enatioselectivities and yields (Scheme 5). Evaluation of the substrate scope was also undertaken and revealed that various heterocycles can be employed, including thiazoles, oxazoles, pyrimidines, triazines, and quinolones (some of the examples are presented in Scheme 5). Unfortunately, no product was observed under the optimised conditions when using the less reactive, but perhaps most important class of heterocycles, namely alkenyl-pyridines. ${ }^{[38]}$ Further optimisation of the reaction conditions eventually revealed that alkenyl pyridines require a stronger LA, such as TMSOTf, and a different solvent, namely dichloromethane. Following these changes we obtained various pyridine-derived addition products with good yields and excellent enantioselectivities (Scheme 5). ${ }^{39]}$

\subsection{Copper(I)-catalysed Enantioselective Addition of Grignard Reagents to $\alpha$, $\beta$-unsatu- rated Carboxamides}

Chiral $\mathrm{Cu}(\mathrm{I})$-catalysts permit additions of hard organometallics to various reactive Michael acceptors, $[27,34-36]$ but despite almost 80 years of intensive research, catalytic additions to simple unactivated $\alpha, \beta$-unsaturated amides are not possible. ${ }^{[40]}$ The complications inhibiting the development of efficient and stereoselective alkylations of simple enamides has led to alternative approaches using specific activated amide substrates, or chiral auxiliary strategies. The only reported examples of efficient catalytic ACA to simple amides were confined to Rh-catalysed arylations. ${ }^{[40]}$ The challenges associated with catalytic ACA to $\alpha, \beta$-unsaturated amides are related to the sluggish resonance activation of the olefin moiety via the adjacent carboxamide, ${ }^{[41]}$ which makes it the least electron-deficient carboxylic acid derivative, arguably even less than alkenyl-heteroarenes. The resonance stabilisation that hampers the reactivity of

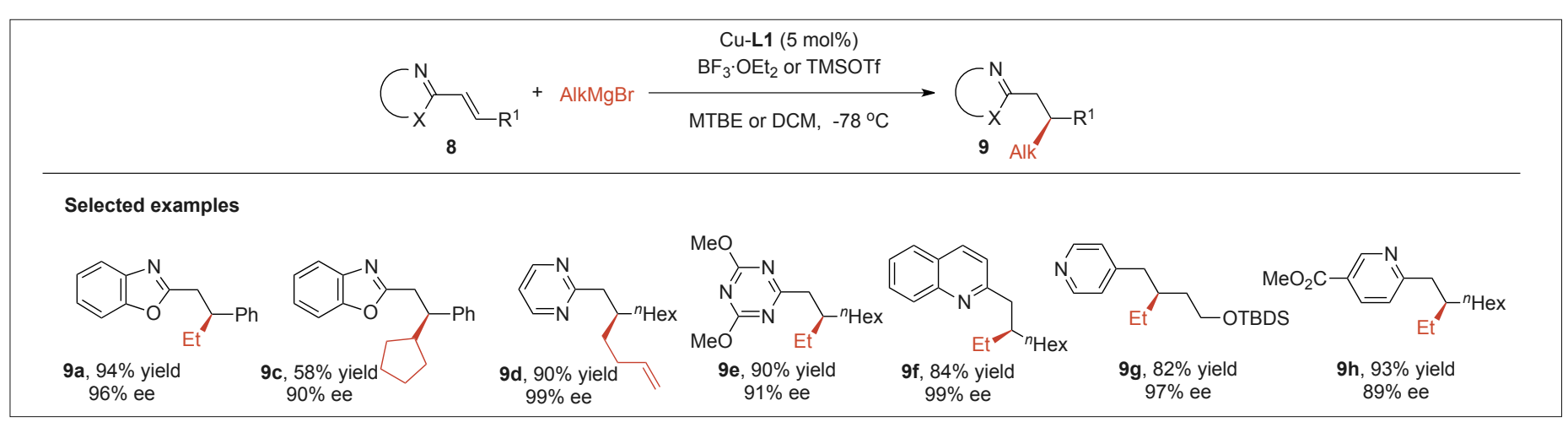

Scheme 5. Representative examples for Lewis acid-promoted copper(I)-L1 catalyzed asymmetric addition of Grignard reagents to alkenyl- $N$-heteroarenes. 
enamides simultaneously grants the amidic carbonyl oxygen atom a pronounced Lewis basicity, that could be exploited to activate the enamides in situ. We hypothesised that coordination of a strong LA with the Lewis basic amidic carbonyl oxygen would allow a significant enhancement in the electrophilicity of the adjacent olefinic moiety towards nucleophilic addition. Analogously to the chemistry with alkenyl-heteroarenes, this in turn could allow direct additions of hard nucleophiles, namely Grignard reagents, to simple unactivated enamides without the need for specific substrates, while rendering the reaction enantioselective by using chiral catalysts. ${ }^{[42]}$

The optimisation results revealed that the use of a LA such as $\mathrm{BF}_{3} \cdot \mathrm{OEt}_{2}$ or TMSOTf is indeed crucial for achieving both good yields and enantioselectivities for the addition products (Scheme 6). Interestingly, when carrying out the reaction with activated amide in the absence of a LA the CA product 11d was obtained with a low $35 \%$ ee. However, CA in the presence of $\mathrm{BF}_{3} \cdot \mathrm{OEt}_{2}$ yielded the product with a high ee of $86 \%$, indicating that LA preferentially accelerates the catalytic ACA pathway over the non-catalysed one. This catalytic system tolerates $\alpha, \beta$-unsaturated amides with electronically and sterically different substituents both at the $\beta$-position and on the $N$-atom (for examples see Scheme 6).

Also the scope with respect to Grignard reagents is remarkably broad, as excellent results were obtained with both linear and branched carbon chains. Interestingly, although both boron- and silicon-based LAs enable ACA to almost all tested enamides, TMSOTf generally works best for relatively unreactive and unhindered enamides, while $\mathrm{BF}_{3} \cdot \mathrm{OEt}_{2}$ is the LA of choice for relatively reactive, both hindered and unhindered, enamides. Furthermore, thanks to the presence of LA the challenge of achieving methylation of unsaturated amides also becomes possible (Scheme 6, 11k). Finally, important features of this catalytic system are its scalability (10 g) and tolerance to temperature. For the first time high levels of enantio- and regioselectivity in the ACA of hard organometallics to Michael acceptors are possible at $0{ }^{\circ} \mathrm{C}$ and above.

\section{Conclusions}

The history of LA-promoted synthesis and copper catalysis dates back several decades and although many spectacular and useful transformations have been developed, the full potential of these concepts are still unexplored. In this review we have shown that combining strong LAs, copper catalysis and reactive organometallics allows to overcome major issues in organic chemistry by fine tuning of chemo-, regio- and enan-

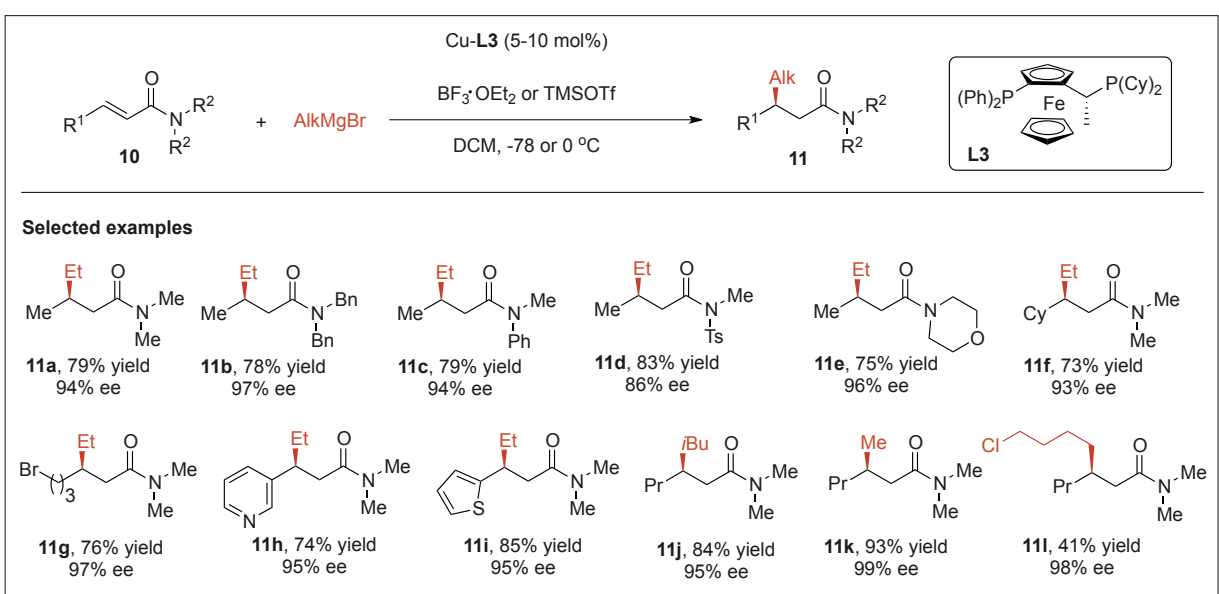

Scheme 6. Representative examples for Lewis acid-enabled copper(I)-L3 catalysed enantioselective alkylation of $\alpha, \beta$-unsaturated carboxamides with Grignard reagents.

tioselectivities in chemical reactions. The synergistic action of these reagent combinations, often considered incompatible, can be a highly powerful tool to solve various issues encountered in synthesis and should be further explored in organic chemistry.

Received: July 27, 2018

[1] H.-J. Federsel, Nat. Rev. 2005, 4, 685 .

[2] 'Asymmetric Catalysis on Industrial Scale: Challenges. Approaches and Solutions', Eds. H.-U. Blaser , H.-J. Federsel, Wiley-VCH, Weinheim, Germany, 2010.

[3] F. Lovering, J. Bikker, C. Humblet, J. Med. Chem. 2009, 52, 67521 .

[4] 'Catalytic Asymmetric Synthesis', Ed. I. Ojima, 3rd Edn., Wiley, Hoboken, New Jersey, 2010.

[5] J. Rong, T. Pellegrini, S. R. Harutyunyan, Chem. Eur. J. 2016, 22, 3558 .

[6] J. F. Collados, R. Solà, S. R. Harutyunyan, B. Maciá, ACS Catalysis 2016, 6, 1952.

[7] A. V. R. Madduri, A. J. Minnaard, S. R. Harutyunyan, Chem. Commun. 2012, 48, 1478.

[8] A. V. R. Madduri, S. R. Harutyunyan, A. J. Minnaard, Angew. Chem. Int. Ed. 2012, 51, 3164.

[9] J. Rong, R. Oost, A. Desmarchelier, A. J. Minnaard, S. R. Harutyunyan, Angew. Chem. Int. Ed. 2015, 54, 3038.

[10] M. Hatano, S. Suzuki, K. Ishihara, J. Am. Chem. Soc. 2006, 128, 9998.

[11] G. Giacomelli, L. Lardicci, R. Santi, J. Org. Chem. 1974, 39, 2736.

[12] 'Lewis Acids in Organic Synthesis', Vol. 1-2, Ed. H. Yamamoto, Wiley-VCH, Weinheim, Germany, 2000

[13] Y. Yamamoto, S. Yamamoto, H. Yatagai, Y. Ishihara, K. Maruyama, J. Org. Chem. 1982, 47, 119.

[14] E. Marcantoni, M. Petrini, 'Lewis Acid Promoted Addition Reactions of Organometallic Compounds', Elsevier, 2014, p. 344.

[15] M. A. Brook, 'Silicon in Organic, Organometallic and Polymer Chemistry', Wiley, New York, 2000.

[16] H.-J. Zhang, D. L. Priebbenow, C. Bolm, Chem. Soc. Rev. 2013, 42, 8540 .

[17] N. Arai, K. Suzuki, S. Sugisaki, H. Sorimachi, T. Ohkuma, Angew. Chem. Int. Ed. 2008, 47, 1770 .

[18] J.-I. Matsuo,Y. Hattori, H. Ishibashi, Org. Lett. 2010, 12, 2294.

[19] V. Cirriez, C. Rasson, T. Hermant, J. Petrignet, J. Díaz Álvarez, K. Robeyns, O. Riant, Angew. Chem. Int. Ed. 2013, 52, 1785.
[20] P. Smirnov, J. Mathew, A. Nijs, E. Katan, M Karni, C. Bolm, Y. Apeloig, I. Marek, Angew. Chem. Int. Ed. 2013, 52, 13717.

[21] G. Gao, X. Bai, F. Li, L. Zheng, Z. Zheng, G. Lai, K. Jiang, F. Li, L. Xu, Tetrahedron Lett. 2012, 53, 2164.

[22] T. Imamoto, N. Takiyama, K. Nakamura, T. Hatajima, Y. Kamiya, J. Am. Chem. Soc. 1989, $111,4392$.

[23] I. B. Sivaev, V. I. Bregadze, Coord. Chem. Rev. 2014, 75,270 .

[24] D. Ameen, T. J. Snape, Med. Chem. Commun. 2013, 4, 893.

[25] D. J. Ramón, M. Yus, Chem. Rev. 2006, 106, 2126.

[26] P. Ortiz, A. M. del Hoyo, S. R. Harutyunyan, Eur. J. Org. Chem. 2015, 1, 72.

[27] G. P. Howell, Org. Process Res. Dev. 2012, 16 , 1258.

[28] D. A. Klumpp, Synlett 2012, 23, 1590.

[29] D. Best, H. W. Lam, J. Org. Chem. 2014, 79, 831.

[30] 'Pharmaceutical Process Development: Current Chemical and Engineering Challenges', Eds. J. A. Blacker, M. T. Williams, Royal Society of Chemistry, 2011

[31] G. Pattison, G. Piraux, H. W. Lam, J. Am. Chem. Soc. 2010, 132, 14373.

[32] A. Saxena, H. W. Lam, Chem. Sci. 2011, 2 , 2326.

[33] L. Rupnicki, A. Saxena, H. W. Lam, J. Am. Chem. Soc. 2009, 131, 10386.

[34] A. Alexakis, N. Krause, S. Woodward, 'CopperCatalysed Asymmetric Synthesis', Wiley-VCH, Weinheim, Germany, 2014.

[35] 'Progress in Enantioselective $\mathrm{Cu}(\mathrm{I})$-catalysed Formation of Stereogenic Centers', Ed. S. R. Harutyunyan, Springer, Heidelberg, 2015.

[36] S. R. Harutyunyan, T. den Hartog, K. Geurts, A J. Minnaard; B. L. Feringa, Chem. Rev. 2008 , 108, 2824.

[37] R. P. Jumde, F. Lanza, M. J. Veenstra, S. R. Harutyunyan, Science 2016, 352, 433.

[38] E. Vitaku, D. T. Smith, J. T. Njardarson, J. Med. Chem. 2014, 57, 10257.

[39] R. P. Jumde, F. Lanza, T. Pellegrini, S. R Harutyunyan Nat. Commun. 2017, 8, 2058.

[40] K. M. Byrd, Beilstein J. Org. Chem. 2015, 11 , 530

[41] A. Greenberg, C. M. Breneman, J. F. Liebman, 'The Amide Linkage: Structural Significance in Chemistry, Biochemistry and Materials Science', Wiley, 2000.

[42] M. Rodríguez-Fernández, X. Yan, J. F. Collados, P. B. White, S. R. Harutyunyan, J. Am. Chem. Soc. 2017, 139, 14224. 